\title{
Group Decision Making Problems in a Linguistic and Dynamic Context
}

\author{
I.J. Pérez, F.J. Cabrerizo and E. Herrera-Viedma
}

\begin{abstract}
The aim of this paper is to present a new model of decision support system for group decision making problems based on a linguistic approach and dynamic sets of alternatives. The model incorporates a mechanism that allows to manage dynamic decision situations in which some information about the problem is not constant in time. We assume that the set of alternatives can change during the decision making process. The model is presented in a mobile and dynamic context where the experts' preferences can be incomplete. The linguistic approach is used to represent both the experts' preferences about the alternatives and the agreement degrees to manage the change of some alternatives. A prototype of such mobile decision support system in which the experts use mobile devices to provide their linguistic preferences at anytime and anywhere has been implemented. In such a way, we provide a new linguistic group decision making framework that is mobile and dynamic.
\end{abstract}

Keywords: Group decision making, mobile internet, decision support system, linguistic approach.

\section{Introduction}

Group Decision Making (GDM) is used to obtain the best solution(s) for a problem according to the information provided by some decision makers. Usually, each decision maker (expert) may approach the decision process from a different angle, but they have a common interest in reaching an agreement on taking the best decision. Concretely, in a GDM problem we have a set of different alternatives to solve the problem and a set of experts which are usually required to provide their preferences about the alternatives by means of a particular preference format.

Several authors have provided interesting results on GDM with the help of fuzzy theory $[4,8,11,13,21$, $26,41,54]$. There are decision situations in which the experts' preferences cannot be assessed precisely in a quantitative form but may be in a qualitative one, and thus, the use of a linguistic approach is necessary $[4,7,25,28,61]$. The linguistic approach is an approximate technique which represents qualitative aspects as linguistic values by means of linguistic variables, that is, variables whose values are not numbers but words or sentences in a natural or artificial language [24].

In this paper we will assume that experts provide their preferences using linguistic preference relations [23]. Other different issue arises when each expert has his/her own experience concerning the problem being studied and they could have some difficulties in giving all their preferences. This may be due to an expert not possessing a precise or sufficient level of knowledge of the problem, or because that expert is unable to discriminate the degree to which some options are better than others. In such situations, experts are forced to provide incomplete linguistic preference relations $[3,14,31,32,34,50]$. Therefore, it is of great importance to provide tools to deal with this lack of knowledge in experts' opinions.

However, GDM is still a difficult process by different reasons as the complexity of real problems, which usually deal with large or dynamic sets of alternatives, or as the lack of clear, complete and timely information and also as the geographical dispersion of experts. A GDM process should be agile so that the experts can share and process information rapidly. Furthermore, there are decision situations where some data of decision process can change through the time. For example, this happens in decision making in geographic context 
in [10], in business [59], in navigation applications [19, 43], in natural resources management [16]. We focus on the configuration data of a GDM problem that could change through time, as for example decision criteria, the members of the group of experts and the alternatives of the initial set of alternatives. Therefore, the structure of a GDM model should be flexible to deal with those changes that could alter the initial configuration of a GDM problem while experts make their decision $[48,53,59]$.

On the other hand, we find that in many domains, the activities of planning and executing a task are disconnected in the sense that they happen at different places or are carried out by different persons. In order to bridge that gap and make sure that models of the planned activity and its actual realization are identical it is desirable to make further use of the mobile technologies [56]. The incorporation of new communication technologies $[42,55]$, besides solving the geographical dispersion of experts problem, extends opportunities for decision making [18], because if communications are improved, decisions will usually be upgraded as well. This improvement is provided by mobile technologies because it is a good way for a continuous communication flow. It allows that experts always have updated and timely information at anywhere and anytime, obtaining in this way an agile decision process by simulating a real discussion meeting.

In this paper we present a new model of decision support system for GDM problems based on a linguistic approach and dynamic sets of alternatives. Experts provide their preferences by means of linguistic preference relations which could be incomplete, i.e., this new GDM model is user-friendly. We consider that the configuration data of the GDM problem with respect to the initial set of alternatives can change through decision process, i.e., this new model presents a dynamic and flexible structure. Furthermore, we implement a prototype of such GDM model using mobile technologies allowing experts to develop the decision process in anywhere and anytime. In such a way, we present a very complete GDM framework that facilitates the development of GDM processes.

In order to do this, the paper is set out as follows. Preliminaries are presented in Section 2. Section 3 defines the new linguistic GDM model. Section 4 presents the prototype of such linguistic GDM model. A case of use is shown in Section 5, and finally, Section 6 draws our conclusions.

\section{Preliminaries}

In the following subsections we present the ordinal fuzzy linguistic approach, GDM problems with incomplete fuzzy linguistic preference relations, and the foundations of mobile technologies.

\subsection{Ordinal fuzzy linguistic approach}

There are situations in which the information cannot be assessed precisely in a quantitative form but may be in a qualitative one. For example, when attempting to qualify phenomena related to human perception, we are often led to use words in natural language instead of numerical values, e.g. when evaluating quality of a football player, terms like good, medium or bad can be used.

The ordinal fuzzy linguistic approach $[22,27]$ is a tool based on the concept of linguistic variable [60] to deal with qualitative assessments. It is a very useful kind of fuzzy linguistic approach because its use simplifies the processes of computing with words as well as linguistic representation aspects of problems. It has proven its usefulness in many problems, e.g., in decision making $[7,11,24,36]$, web quality evaluation $[38,37,39]$, information retrieval $[9,30,35]$, recommender systems [51, 52], evaluation of libraries [12, 44], political analysis [5], etc.

It is defined by considering a finite and totally ordered label set $S=\left\{s_{i}\right\}, i \in\{0, \ldots, g\}$ in the usual sense, i.e., $s_{i} \geq s_{j}$ if $i \geq j$, and with odd cardinality (usually 7 or 9 labels). The mid term represents an assessment of "approximately 0.5 ", and the rest of the terms are placed symmetrically around it. The semantics of the label set is established from the ordered structure of the label set by considering that each label for the pair $\left(s_{i}, s_{g-i}\right)$ is equally informative [24]. For example, we can use the following set of seven labels to represent the linguistic information: $S=\left\{s_{0}=N, s_{1}=V L, s_{2}=L, s_{3}=M, s_{4}=H, s_{5}=V H, s_{6}=P\right\}$, where $\mathrm{N}=$ Null, $\mathrm{VL}=$ Very Low, $\mathrm{L}=$ Low, $\mathrm{M}=$ Medium, $\mathrm{H}=$ Hight, $\mathrm{VH}=$ Very Hight and $\mathrm{P}=$ Perfect. 
In any linguistic modeling we also need some management operators for linguistic information. An advantage of the ordinal fuzzy linguistic modeling is the simplicity and speed of its computational model. It is based on the symbolic computational model [22, 27] and acts by direct computation on labels by taking into account the order of such linguistic assessments in the ordered structure of labels. Usually, the ordinal fuzzy linguistic model for computing with words is defined by establishing i) a negation operator, ii) comparison operators based on the ordered structure of linguistic terms, and iii) adequate aggregation operators of ordinal fuzzy linguistic information. In most ordinal fuzzy linguistic approaches the negation operator is defined from the semantics associated to the linguistic terms as $N E G\left(s_{i}\right)=s_{j} \mid j=g-i$; and there are defined two comparison operators of linguistic terms:

1. Maximization operator: $M A X\left(s_{i}, s_{j}\right)=s_{i}$ if $s_{i} \geq s_{j} ;$ and

2. Minimization operator: $\operatorname{MIN}\left(s_{i}, s_{j}\right)=s_{i}$ if $s_{i} \leq s_{j}$.

Using these operators it is possible to define automatic and symbolic aggregation operators of linguistic information, as for example the LOWA operator [27] and the LWA operator [22]. Specifically, we will use the LOWA operator.

\subsection{GDM problems with incomplete fuzzy linguistic preference relations}

In a GDM problem we have a finite set of feasible alternatives $X=\left\{x_{1}, x_{2}, \ldots, x_{n}\right\},(n \geq 2)$ and the question is to find the best alternatives according to the preferences given by a set of experts, $E=$ $\left\{e_{1}, e_{2}, \ldots, e_{m}\right\},(m \geq 2)$.

A usual resolution method for a GDM problem is composed of two different processes [28, 29] (see Figure 1):

1. Consensus process: Clearly, in any decision process, it is preferable that the experts reach a high degree of consensus on the solution set of alternatives. Thus, this process refers to how to obtain the maximum degree of consensus or agreement among the experts on the solution alternatives.

2. Selection process: This process consists in how to obtain the solution set of alternatives from the opinions on the alternatives given by the experts.

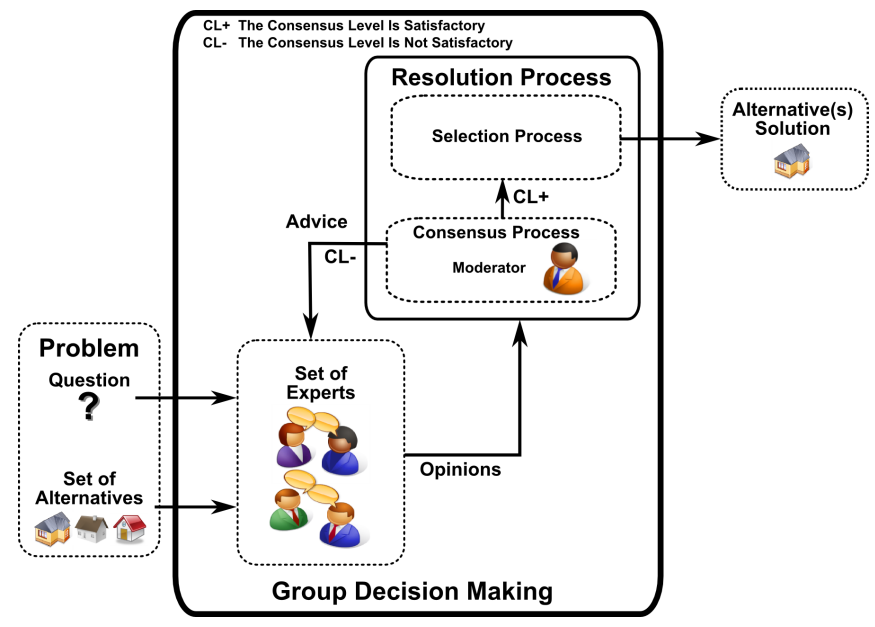

Figure 1: Resolution process of a GDM

In a GDM problem the experts can present their opinions using different elements of preference representation (preference orderings, utility functions or preference relations) [15], but in this paper, we assume that the experts give their preferences using incomplete fuzzy linguistic preference relations. 
Definition 1 A Fuzzy linguistic Preference Relation (FLPR) $P^{h}$ given by an expert $e_{h}$ is a fuzzy set defined on the product set $X \times X$, that is characterized by a linguistic membership function

$$
\mu_{P^{h}}: X \times X \longrightarrow S
$$

where the value $\mu_{P^{h}}\left(x_{i}, x_{k}\right)=p_{i k}^{h}$ is interpreted as the linguistic preference degree of the alternative $x_{i}$ over $x_{k}$ for the expert $e_{h}$.

Definition 2 A FLPR $P^{h}$ is complete when $\forall\left(x_{i}, x_{k}\right) \in(X \times X)$ the expert $e_{h}$ has provided only one linguistic preference degree $p_{i k}^{h} \in S$.

Definition 3 A FLPR $P^{h}$ is incomplete when $\exists\left(x_{i}, x_{k}\right) \in(X \times X)$ such that the expert $e_{h}$ has provided no linguistic preference degree $p_{i k}^{h} \in S$

\subsection{Mobile technologies and GDM}

With the fast increase of the new technologies usage $[42,55]$ and the new services that are offered, the impact in the society of the mobile communication devices is much bigger. Moreover, the growing penetration of mobile devices and the recent technological innovation in the wireless technology field have changed the old wired Internet world to the new wireless mobile Internet world, as known as M-Internet [17, 46, 57].

This recent massive use of wireless technology has strongly modified the organization and management of work and has made critical to gather and estimate a set of decision problem data and to share them among experts in real-time [49].

Nowadays, organizations have moved from face-to-face group environments to virtual group environment using communication technologies and tools to coordinate and share information with other people. The main objective of these new approaches is that the members of the group could work in an ideal way no matter where they are, having all the necessary information to take the most guessed right decisions. Using the mobile technologies, besides increasing the productivity and the satisfaction of the user, allows to save the operational costs of having to bring together to the complete group in the same place at the same time.

To support the new generation of decision makers and to add real-time process in the GDM field, many authors have proposed to develop decision support systems based on mobile technologies [18, 20, 58]. Similarly, we propose to incorporate mobile technologies in our DSS obtaining a Mobile DSS (MDSS). Using such a technologies should enable users to maximize the advantages and minimize the drawbacks of DSSs.

While DSSs have typically been associated with desktop systems and involve considerable processing, the development of new compact and mobile technologies provides new opportunities to develop this kind of DSSs over M-Internet $[6,42,55]$. The incorporation of mobile technologies on the GDM process is based on the supposition that if the communications are improved the decisions will be upgraded $[18,40,42,55,58]$ . If the communications are improved, then the discussion can be focused on the problem and less time is lost with unimportant issues. This saved time can be used to do an exhaustive analysis of the problem and to obtain a better problem definition. This time also could be used to identify more alternatives that can be solutions of the problem.

The language used to compute interfaces and the form of human computer interaction are key obstacles to provide this sort of mobile decision support. The physical limitations and style of use of mobile devices requires a specialized form of decision support system. The design of the decision support models and their customization to particular tasks is more easily done using less restrictive, declarative styles of description [18]. Mobile computing requires solutions for service discovery in a dynamic environment, establishment of data communication channels between devices and service providers, development tools that allow the integration of devices and distributed resources, and security, such as confidentiality of data and authentication [47]. In Section 4, we describe an architecture, which helps to overcome these obstacles.

The Mobile Web mainly uses lightweight pages written in Extensible Hypertext or Wireless Markup Language (XHTML) or (WML), to deliver content to mobile devices. However, new tools such as Macromedias Flash Lite or Sun's J2ME enable the production of richer user interfaces customized for mobile devices. To support these requirements, we develop J2ME applications for mobile devices that implement the new GDM 
models using the best architecture for each model. These applications can be installed on any mobile device equipped whit a Java Virtual Machine (JVM).

\section{A new model of linguistic GDM based on mobile technologies and dynamic information}

In this section we present a new GDM model that incorporates a mechanism to manage some dynamic information that might change during the decision process, in particular, the alternatives to be analyzed. Furthermore, the model is specifically designed to use mobile devices as the main communication tool and we allow that the experts use incomplete FLPRs to provide their preferences. In such a way, GDM processes could be developed at anytime and anywhere and we can simulate with more accuracy level the real processes of human decision making which are developed in dynamic environments as the Web, financial investment, health, navigation, natural resources management and so on.

This new linguistic, dynamic and mobile GDM model is composed of the following five processes (see Figure 2):

1. An estimation process to complete the incomplete FLPRs.

2. A selection process to obtain a temporary solution.

3. A consensus process to measure the agreement degree.

4. A managing process of dynamic information to deal with the dynamic set of alternatives.

5. A feedback process to help experts in the consensus reaching process.

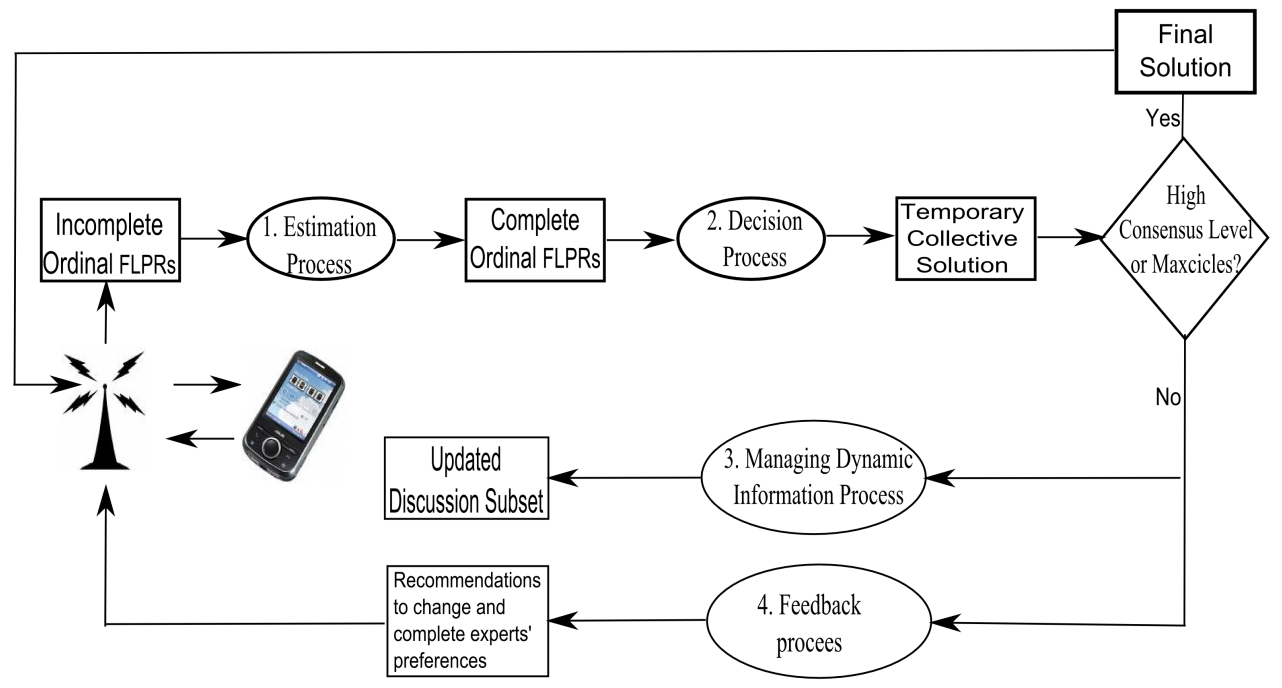

Figure 2: Structure of the linguistic, dynamic and mobile GDM model

\subsection{Estimation process}

In [3] we develop an additive consistency based estimation process of missing values to deal with incomplete FLPRs defined in a 2-tuple linguistic context. In this paper we adapt it to deal with incomplete FLPRs defined in an ordinal linguistic context. 
To deal with incomplete FLPRs we need to define the following sets [31]:

$$
\begin{aligned}
& A=\{(i, j) \mid i, j \in\{1, \ldots, n\} \wedge i \neq j\} \\
& M V^{h}=\left\{(i, j) \in A \mid p_{i j}^{h} \text { is unknown }\right\} \\
& E V^{h}=A \backslash M V^{h}
\end{aligned}
$$

where $M V^{h}$ is the set of pairs of alternatives whose preference degrees are not given by expert $e_{h}$ and $E V^{h}$ is the set of pairs of alternatives whose preference degrees are given by the expert $e_{h}$. We do not take into account the preference value of one alternative over itself as this is always assumed to be equal to $s_{g / 2}$.

Then, the subset of missing values that could be estimated in step $t$ of the process, called $E M V_{t}^{h}$ (estimated missing values), is defined as follows:

$$
E M V_{t}^{h}=\left\{(i, k) \in M V^{h} \backslash \bigcup_{l=0}^{t-1} E M V_{l}^{h} \mid i \neq k \wedge \exists j \in\left\{H_{i k}^{t 1} \cup H_{i k}^{t 2} \cup H_{i k}^{t 3}\right\}\right\},
$$

with

$$
\begin{aligned}
& H_{i k}^{t 1}=\left\{j \mid(i, j),(j, k) \in\left\{E V \bigcup_{l=0}^{t-1} E M V_{l}\right\}\right\} \\
& H_{i k}^{t 2}=\left\{j \mid(j, i),(j, k) \in\left\{E V \bigcup_{l=0}^{t-1} E M V_{l}\right\}\right\} \\
& H_{i k}^{t 3}=\left\{j \mid(i, j),(k, j) \in\left\{E V \bigcup_{l=0}^{t-1} E M V_{l}\right\}\right\}
\end{aligned}
$$

and $E M V_{0}^{h}=\emptyset$ (by definition). When $E M V_{\text {maxIter }}^{h}=\emptyset$, with maxIter $>0$, the procedure will stop as there will not be any more missing values to be estimated. Furthermore, if $\bigcup_{l=0}^{\max I t e r} E M V_{l}^{h}=M V^{h}$, then all missing values are estimated, and, consequently, the procedure is said to be successful in the completion of the incomplete FLPR.

In iteration t, to estimate a particular value $p_{i k}^{h}$ with $(i, k) \in E M V_{t}^{h}$, the following function estimate_p $(h, i, k)$ is proposed:

$$
\begin{aligned}
& \text { function estimate_p(h,i,k) } \\
& \text { 1) }\left(c p_{i k}^{h}\right)^{1}=\left(s_{0}\right),\left(c p_{i k}^{h}\right)^{2}=\left(s_{0}\right),\left(c p_{i k}^{h}\right)^{3}=\left(s_{0}\right), \mathcal{K}=0 \\
& 2) \text { if } \#\left(H_{i k}^{t 1}\right) \neq 0 \text {, then }\left(c p_{i k}^{h}\right)^{1}=\frac{\sum_{j \in H_{i k}^{t 1}}\left(c p_{i k}^{h}\right)^{j 1}}{\#\left(H_{i k}^{t 1}\right)}, \mathcal{K}++ \\
& 3) \text { if } \#\left(H_{i k}^{t 2}\right) \neq 0 \text {, then }\left(c p_{i k}^{h}\right)^{2}=\frac{\sum_{j \in H_{i k}^{t 2}}\left(c p_{i k}^{h}\right)^{j 2}}{\#\left(H_{i k}^{t 2}\right)}, \mathcal{K}++ \\
& 4) \text { if } \#\left(H_{i k}^{t 3}\right) \neq 0 \text {, then }\left(c p_{i k}^{h}\right)^{3}=\frac{\sum_{j \in H_{i k}^{t 3}}\left(c p_{i k}^{h}\right)^{j 3}}{\#\left(H_{i k}^{t 3}\right)}, \mathcal{K}++ \\
& \text { 5) } c p_{i k}^{h}=s_{q} \in S \mid q=\operatorname{round}\left(\frac{\left(c p_{i k}^{h}\right)^{1}+\left(c p_{i k}^{h}\right)^{2}+\left(c p_{i k}^{h}\right)^{3}}{\mathcal{K}}\right) \\
& \text { end function }
\end{aligned}
$$

being

$$
\begin{aligned}
\text { - }\left(c p_{i k}^{h}\right)^{j 1} & =\left\{\begin{array}{ll}
g & \text { if } v^{\prime} \geq \frac{3}{2} g \\
0 & \text { if } v^{\prime}<0 \\
v^{\prime} & \text { othercase }
\end{array} \text { with } v^{\prime}=I\left(p_{i j}^{h}\right)+I\left(p_{j k}^{h}\right)-I\left(s_{g / 2}\right) .\right. \\
\text { - }\left(c p_{i k}^{h}\right)^{j 2} & =\left\{\begin{array}{cc}
g & \text { if } v^{\prime} \geq \frac{3}{2} g \\
0 & \text { if } v^{\prime}<0 \\
v^{\prime} & \text { othercase }
\end{array} \text { with } v^{\prime}=I\left(p_{j k}^{h}\right)-I\left(p_{j i}^{h}\right)+I\left(s_{g / 2}\right) .\right.
\end{aligned}
$$


- $\left(c p_{i k}^{h}\right)^{j 3}=\left\{\begin{array}{l}g \quad \text { if } v^{\prime} \geq \frac{3}{2} g \\ 0 \quad \text { if } v^{\prime}<0 \\ v^{\prime} \quad \text { othercase }\end{array}\right.$ with $v^{\prime}=I\left(p_{i j}^{h}\right)-I\left(p_{k j}^{h}\right)+I\left(s_{g / 2}\right)$.

- and $I: S \rightarrow\{0, \ldots, g\} \mid I\left(s_{p}\right)=p \forall s_{p} \in S$.

Then, the complete iterative estimation procedure is the following:

\section{ITERATIVE ESTIMATION PROCEDURE}

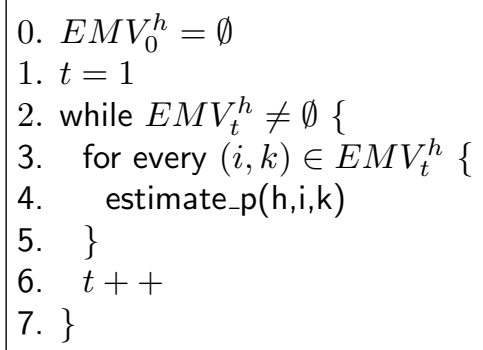

In [3] was demonstrated that an incomplete FLPR can be completed if a set of $n-1$ non-leading diagonal preference values, where each one of the alternatives is compared at least once, is known.

\subsection{Selection process}

The selection has two different phases [26]:

1. Aggregation:

This phase defines a collective preference relation, $P^{c}=\left(p_{i j}^{c}\right)$, obtained by means of the aggregation of all individual linguistic preference relations $\left\{P^{1}, P^{2}, \ldots, P^{m}\right\}$. It indicates the global preference between every pair of alternatives according to the majority of experts' opinions. The aggregation is carried out by means of a LOWA operator $\phi_{Q}$ guided by a fuzzy linguistic non-decreasing quantifier $Q$ [27]:

$$
p_{i j}^{c}=\phi_{Q}\left(p_{i j}^{1}, \ldots, p_{i j}^{m}\right)
$$

2. Exploitation:

This phase transforms the global information about the alternatives into a global ranking of them, from which the set of solution alternatives is obtained. The global ranking is obtained applying these two choice degrees of alternatives on the collective preference relation [23]:

(a) $Q G D D_{i}$ : This quantifier guided dominance degree quantifies the dominance that one alternative $x_{i}$ has over all the others in a fuzzy majority sense:

$$
Q G D D_{i}=\phi_{Q}\left(p_{i 1}^{c}, p_{i 2}^{c}, \ldots, p_{i(i-1)}^{c}, p_{i(i+1)}^{c}, \ldots, p_{i n}^{c}\right)
$$

This measure allows us to define the set of non-dominated alternatives with maximum linguistic dominance degree:

$$
X^{Q G D D}=\left\{x_{i} \in X \mid Q G D D_{i}=\sup _{x_{j} \in X} Q G D D_{j}\right\}
$$

(b) $Q G N D D_{i}$ : This quantifier guided non-dominance degree gives the degree in which each alternative $x_{i}$ is not dominated by a fuzzy majority of the remaining alternatives:

$$
Q G N D D_{i}=\phi_{Q}\left(N E G\left(p_{1 i}^{s}\right), N E G\left(p_{2 i}^{s}\right), \ldots, N E G\left(p_{(i-1) i}^{s}\right), N E G\left(p_{(i+1) i}^{s}\right), \ldots, N E G\left(p_{n i}^{s}\right)\right)
$$

where 


$$
p_{i j}^{s}= \begin{cases}s_{0} & \text { if } p_{i j}^{c}<p_{j i}^{c} \\ s_{I\left(p_{i j}^{c}\right)-I\left(p_{j i}^{c}\right)} & \text { if } p_{i j}^{c} \geq p_{j i}^{c}\end{cases}
$$

represents the degree in which $x_{i}$ is strictly dominated by $x_{j}$. The set of of non-dominated alternatives with maximum linguistic non-dominance degree is

$$
X^{Q G N D D}=\left\{x_{i} \in X \mid Q G N D D_{i}=\sup _{x_{j} \in X} Q G N D D_{j}\right\}
$$

Finally, the solution $X_{\text {sol }}$ is obtained as:

$$
X_{\text {sol }}=X^{Q G D D} \cap X^{Q G N D D} .
$$

\subsection{Consensus process}

we assume that the consensus as a measurable parameter whose highest value corresponds to unanimity and lowest one to complete disagreement. We use some consensus degrees to measure the current level of consensus in the decision process. They are given at three different levels [28, 29, 45]: pairs of alternatives, alternatives and relations. The computation of the consensus degrees is carried out as follows:

1. For each pair of experts, $e_{i}, e_{j}(i<j)$, a similarity matrix, $S M^{i j}=\left(s m_{l k}^{i j}\right)$, is defined where

$$
s m_{l k}^{i j}=1-\frac{\left|I\left(p_{l k}^{i}\right)-I\left(p_{l k}^{j}\right)\right|}{g} .
$$

2. A consensus matrix, $C M$, is calculated by aggregating all the similarity matrices using the arithmetic mean as the aggregation function $\phi$ :

$$
c m_{l k}=\phi\left(s m_{l k}^{12}, s m_{l k}^{13}, \ldots, s m_{l k}^{1 m}, s m_{l k}^{23}, \ldots, s m_{l k}^{(n-1) n},\right) .
$$

3. Once the consensus matrix, $C M$, is computed, we proceed to calculate the consensus degrees:

(a) Level 1. Consensus degree on pairs of alternatives, $c p_{l k}$. It measures the agreement on the pair of alternatives $\left(x_{l}, x_{k}\right)$ amongst all the experts.

$$
c p_{l k}=c m_{l k} .
$$

(b) Level 2. Consensus degree on alternatives, $\mathrm{ca}_{l}$. It measures the agreement on an alternative $x_{l}$ amongst all the experts.

$$
c a_{l}=\frac{\sum_{k=1}^{n} c p_{l k}}{n} .
$$

(c) Level 3. Consensus degree on the relation, $\mathrm{cr}$. It measures the global consensus degree amongst the experts' opinions.

$$
c r=\frac{\sum_{l=1}^{n} c a_{l}}{n} .
$$

Initially, in this consensus model we consider that in any nontrivial GDM problem the experts disagree in their opinions so that decision has to be viewed as an iterative process. This means that agreement is obtained only after some rounds of consultation. In each round, we calculate the consensus measures and check the current agreement existing among experts using $c r$. 


\subsection{Managing process of dynamic information}

In the real world we find many dynamic decision frameworks, as health, financial investment, military operations, Web, navigation, natural resources management and so on. In such cases, due to different reasons, some information of the problem could vary through decision process. Thus, a model of decision making should present a flexible and adaptive structure to include those changes that could happen through decision process so that we can constantly revise our decision and the parameters of the problem.

Classical GDM models are defined in a static framework. In order to make the decision making process more realistic, we provide a new tool to deal with dynamic parameters in decision making, as for example the set of alternatives or the group of experts. As aforementioned, in this paper we focus on the changes produced in the set of alternatives because it could depend on dynamical external factors like the traffic $[19,43]$, or the meteorological conditions [16], and so on, and this kind of change is more usual. In such a way, we consider dynamic decision problems in which, at every stage of the process, the discussion is centered on different alternatives.

We define a method which allows us to introduce new alternatives in the discussion process. Firstly, the system identifies those new alternatives to include in the set of discussion alternatives and the worst alternatives to eliminate. And then, the system asks experts their opinion about such changes, i.e., if they agree or not.

To identify the new alternatives we can have two particular cases: (see Figures 3 and 4 )

- This first case happens when a good new alternative appears in the set because some dynamic external factors changed during the decision process, and this new alternative deserves to be in the discussion subset. Before including the new alternative in the discussion subset, the system has to identify the worst alternative of the current discussion subset. To find this bad alternative $x_{i}$ we compare the dominance and non dominance degrees $Q G D D_{i}$ and $Q G N D D_{i}$ of all the alternatives, and choose the less evaluated as the worst alternative.

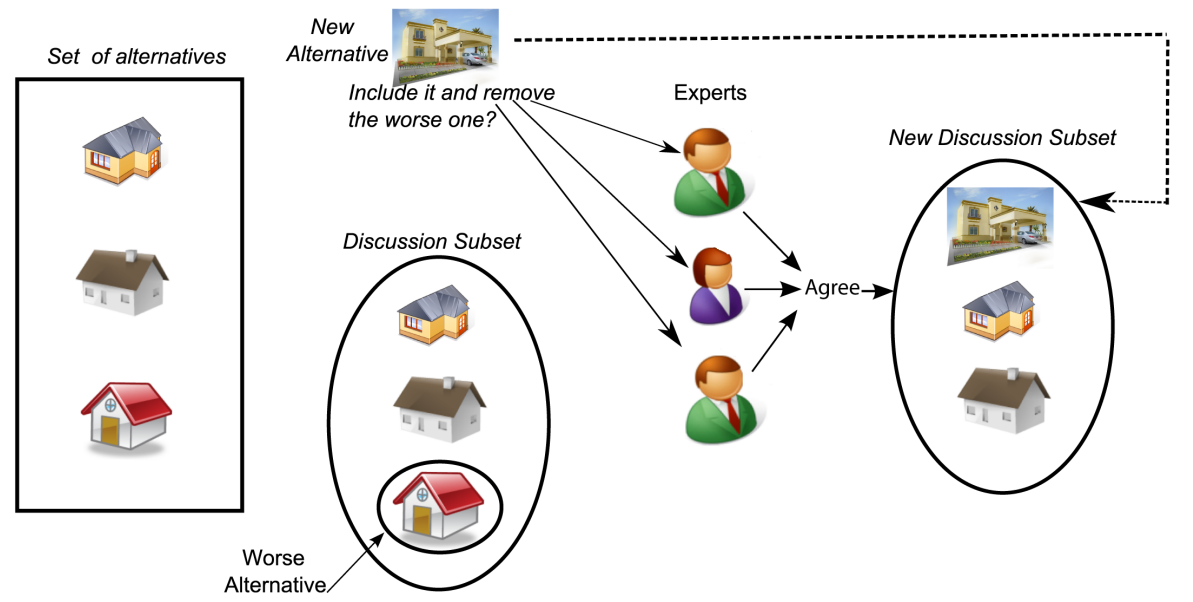

Figure 3: Dynamic choice process of alternatives: case 1

- This second case is when we observe that an alternative $x_{i}$ always receives low dominance and non dominance degrees $Q G D D_{i}$ and $Q G N D D_{i}$ due to the changes of the some dynamic external factors during the decision process. Then we could decide to substitute it by another alternative of the initial set of alternatives that was not included in the discussion set of alternatives. This strategy of replacement is commonly used when there is a big set of possible alternatives and they can not be 
evaluated at the same time. So, we can decide to replace the bad alternatives in the discussion subset in order to evaluate a major number of alternatives. The new alternative to be considered is obtained from the initial list of alternatives that were not included in the discussion subset initially, but now they can be used to replace a bad alternative.

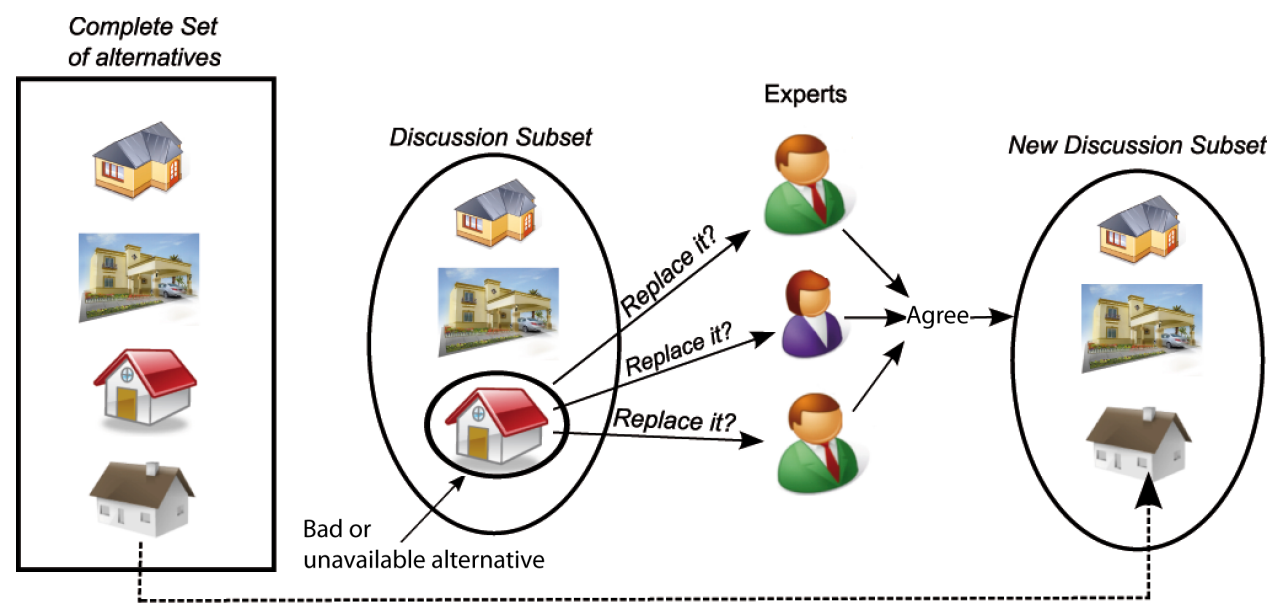

Figure 4: Dynamic choice process of alternatives: case 2

Once the alternatives to be interchanged have been identified, the system gives experts the option to accept or decline the proposed changes. They must provide their their degrees of agreement with the proposed changes using a set of linguistic assessments, as for example:

\{Completely Agree, Agree, Nor Agree/Nor Disagree, Disagree, Completely Disagree\}.

At this point, to avoid stagnation a maxTime threshold is established.

Then, we aggregate degrees of agreement provided by experts using the LOWA operator [27]). If we obtain a high degree of agreement (more than nor agree/nor disagree) then the system removes the bad alternative from the discussion subset of alternatives and the new one is incorporated into this discussion subset.

\subsection{Feedback process}

We apply a feedback mechanism to guide the change of the experts' opinions. This mechanism is able to substitute the moderator's actions in the consensus reaching process. It helps experts to change their preferences and to complete their missing values. The main problem for the feedback mechanism is how to find a way of making individual positions converge and, therefore, how to support the experts in obtaining and agreeing with a particular solution [33].

When the consensus measure $c_{r}$ has not reached the required consensus level (CL) and the number of rounds has not reached a maximum number of iterations (defined prior to the beginning of the decision process) (MAXCYCLE), the experts' opinions must be modified. To do that, we compute others consensus measures, called proximity measures [28], which allows us to build a feedback mechanism so that experts change their opinions and narrow their positions $[31,45]$.

\subsubsection{Computation of proximity measures}

These measures evaluate the agreement between the individual experts' opinions and the group opinion. To compute them for each expert, we need to use the collective FLPR, $P^{c}=\left(p_{l k}^{c}\right)$, calculated previously. 
1. For each expert, $e_{h}$, a proximity matrix, $P M^{h}=\left(p m_{l k}^{h}\right)$, is obtained where

$$
p m_{l k}^{h}=1-\frac{\left|I\left(p_{l k}^{h}\right)-I\left(p_{l k}^{c}\right)\right|}{g} .
$$

2. Computation of proximity measures at three different levels:

(a) Level 1. Proximity measure on pairs of alternatives, $p p_{l k}^{h}$. It measures the proximity between the preferences on each pair of alternatives of the expert $e_{h}$ and the group.

$$
p p_{l k}^{h}=p m_{l k}^{h} .
$$

(b) Level 2. Proximity measure on alternatives, $p a_{l}^{h}$. It measures the proximity between the preferences on each alternative $x_{l}$ of the expert $e_{h}$ and the group.

$$
p a_{l}^{h}=\frac{\sum_{k=1}^{n} p p_{l k}^{h}}{n} .
$$

(c) Level 3. Proximity measure on the relation, $\mathrm{pr}^{h}$. It measures the global proximity between the preferences of each expert $e_{h}$ and the group.

$$
p r^{h}=\frac{\sum_{l=1}^{n} p a_{l}^{h}}{n}
$$

\subsubsection{Production of advice}

The production of advice to achieve a solution with the highest possible degree of consensus is carried out in two phases: Identification phase and Recommendation phase.

1. Identification phase. We must identify the experts, alternatives and pairs of alternatives that are contributing less to reach a high degree of consensus.

(a) Identification of experts. We identify the set of experts, EXPCH, that should receive advice on how to change some of their preference values:

$$
E X P C H=\left\{h \mid p r^{h}<\gamma\right\}
$$

Where $\gamma$ is the minimum proximity level required for the expert to be noted to change.

(b) Identification of alternatives. We identify the alternatives whose associated assessments should be taken into account by the above experts in the change process of their preferences;

$$
A L T_{h}=\left\{x_{l} \in X \mid p a_{l}^{h}<\gamma \wedge h \in E X P C H\right\}
$$

(c) Identification of pairs of alternatives. In this step we identify the particular pairs of alternatives $\left(x_{l}, x_{k}\right)$ whose respective assessments $p_{l k}^{h}$ the expert $e_{h}$ should change.

$$
P A L T_{h}=\left\{\left(x_{l}, x_{k}\right) \mid p p_{l k}^{h}<\gamma \wedge x_{l} \in A L T_{h} \wedge h \in E X P C H\right\}
$$

2. Recommendation phase. In this phase we recommend expert changes of their preferences according to two kinds of rules:

(a) Rules to change the opinions. We must find out the direction of change to be applied to the preference assessment $p_{l k}^{h}$, with $\left(x_{l}, x_{k}\right) \in P A L T_{h}$. To do this, we define the following two direction rules. It is worth to note that if one of the alternatives of the pair $\left(x_{l}, x_{k}\right)$ has been replaced in the managing process of dynamic information, that pair has to be removed from the set $P A L T_{h}$, as there is not need to provide rules for alternatives that are being removed from the discussion subset. 
- If $p_{l k}^{h}>p_{l k}^{c}$, the expert $e_{h}$ should decrease the assessment associated to the pair of alternatives $\left(x_{l}, x_{k}\right)$.

- If $p_{l k}^{h}<p_{l k}^{c}$, the expert $e_{h}$ should increase the assessment associated to the pair of alternatives $\left(x_{l}, x_{k}\right)$.

(b) Rules to complete missing values. Additionally, the feedback process must provide rules for missing preferences values. Thus, the lack of information decreases and in this way better solutions can be obtained. To do so, it has to take into account all missing values that were not provided by the experts and were calculated at the estimation process. The advice generated to complete the preferences is the following:

- "You should provide a value for $p_{l k}^{h}$ near to $c p_{l k}^{h}$ ".

Furthermore, in order to avoid stagnation and give more dynamism and speed to the consensus process, we do not have to wait for all experts who have received recommendations of change provide new preferences: it is enough that a significant number of experts, different for each problem, send their changed preferences to begin the new round of consensus process after a minimum time threshold has been surpassed.

Obviously, the decision process will depend on the size of the group of experts as well as on the size of the set of alternatives, so that when these sizes are small and when opinions are homogeneous, the required consensus level is easier to obtain. This process has been designed to converge to a final solution with a high consensus degree.

\section{Prototype of the new linguistic GDM model}

Here we present the implemented prototype of the linguistic GDM model, explaining and the communication and work flow that summarizes the functions of this system.

The most used architecture for mobile devices is the "Client/Server" architecture (see Figure 5), where the client is a mobile device. The client/server paradigm is founded on the concept that clients (such as personal computers, or mobile devices) and servers (computers) are both connected by a network enabling servers to provide different services for the clients. When a client sends a request to a server, this server processes the request and sends a response back to client.

In addition, we can currently identify two approaches to mobile deployments: thick-client and thin-client.

- Thin-client architectures rely entirely on Web technologies to deliver mobile applications. No additional technology investment is required, and there is no risk of client-side software becoming obsolete. There is no need for additional servers, and no unique client-side software or upgrade costs. Its main drawback is that all content cannot be easily delivered to all browsers.

- Thick-client deployments run special software on each type of mobile device, fed by special servers that manage the interactions with those devices. The client-side software controls how content is displayed. This was an important factor in the early days of mobile browsers, when each device displayed content differently.

We have chosen a thick-client model for our implementation. This allows us to use the software in all the mobile devices without taking into account the kind of browser. Furthermore, the technologies that we have used to implement the prototype comprise Java and Java Midlets for the client software, PHP for the server functions and MySQL for the database management.

According to the model of linguistic GDM model defined in the previous section, the prototype allows user to send his/her preferences by means of a mobile device, and the system returns to the experts the final solution or recommendations to increase the consensus levels, depending of the status of the decision process. An important aspect is that the user-system interaction can be done anytime and anywhere which facilitates expert's participation and the resolution of the decision process.

In what follows, we describe the client and server of the prototype in detail. 


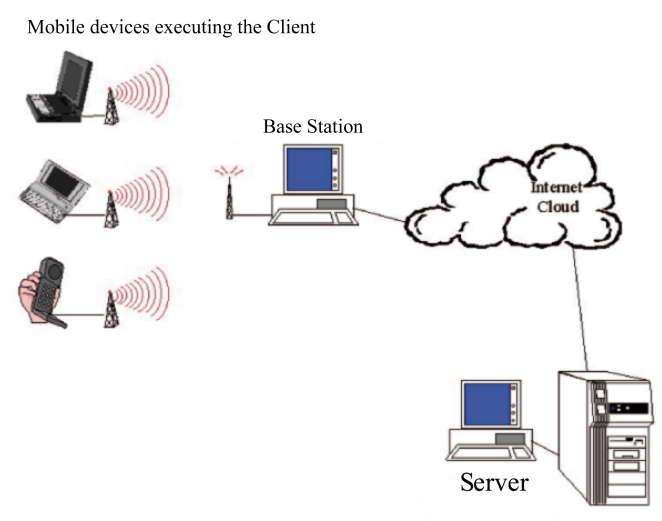

Figure 5: "Client/Server" architecture

\subsection{Client}

The client software shows the next seven interfaces to the experts:

- Authentication: The device asks a user and a password to access the system (see Figure 6a).

- Connection: The device must be connected to the network to send/receive information to the server(see Figure 6b).

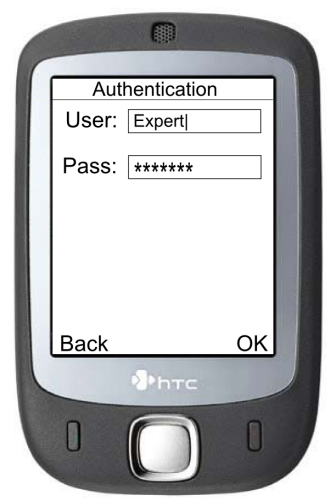

(a) Authentication

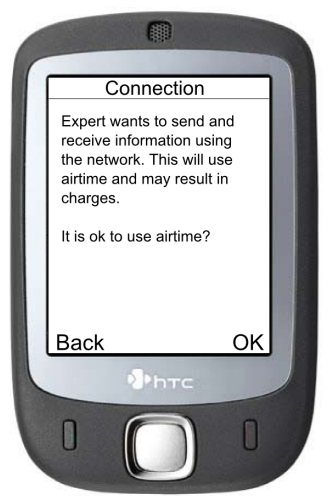

(b) Connection

Figure 6

- Problem description: When a decision process is started, the device shows to the experts a brief description about the problem and the discussion subset of alternatives (see Figure 7a).

- Insertion of preferences: The device will have a specific interface to insert the linguistic preferences using a set of labels (see Figure $7 \mathrm{~b}$ ). To introduce or change the preferences on the interface, the user has to use the keys of the device.

- Swap of Alternatives: When a new alternative appears in the environment of the problem because some dynamic external factors have changed and this alternative deserves to be a member of the discussion 


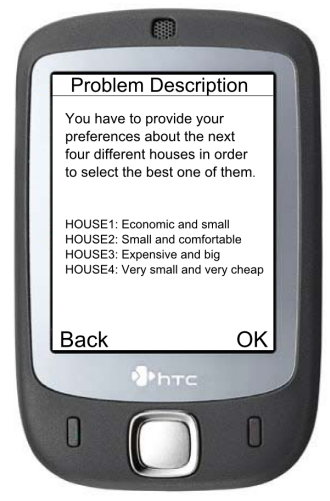

(a) Problem description (b) Insertion Of prefer-

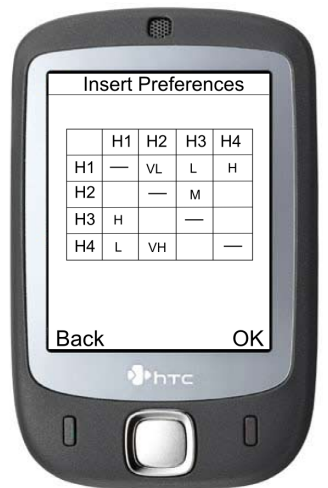

ences

Figure 7

subset or when an alternative have a low dominance degree to the current temporary solution of consensus, the system asks the experts if they want to modify the discussion subset by swapping these alternatives. The experts can assess if they agree to swap the alternatives sending their answer to the question received (Figure 8a). The user can select the chosen degree by using the cursor key of the device.

- Feedback: When opinions should be modified, the device shows experts the recommendations and allows experts to send their new preferences. This system also shows the advice generated to complete the missing values at the last stage (see Figure $8 \mathrm{~b}$ ).

- Output: At the end of the decision process, the device will show the set of solution alternatives as an ordered set of alternatives marking the most relevant ones (see Figure 8c).

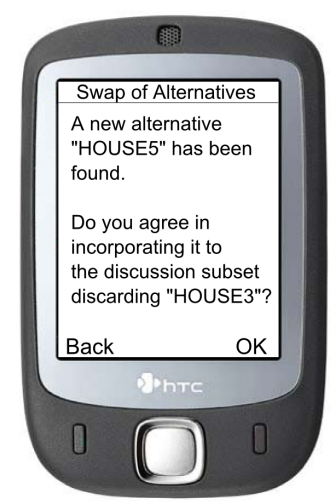

(a) Swap of alternatives

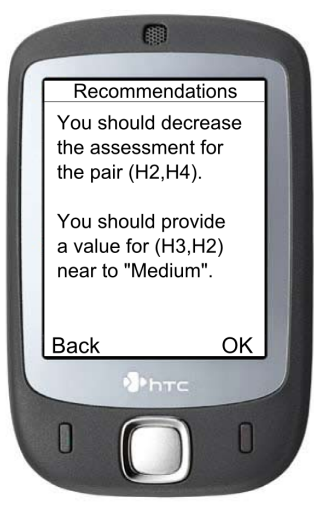

(b) Recommendations

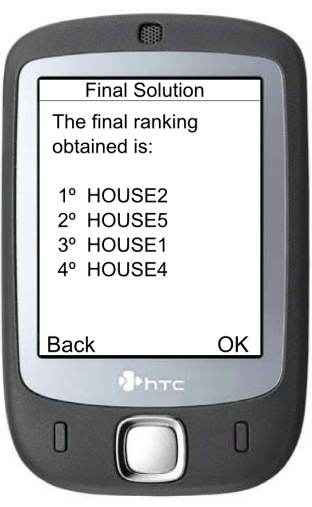

(c) Final solution

Figure 8

On the technical side of the development of the client part, it is worth to note that the client application complies with the MIDP 2.0 specifications [1] and that the J2ME Wireless Toolkit 2.2 [2] provided by SUN was used in the development phase. This wireless toolkit is a set of tools that provide J2ME developers with some emulation environments, documentation, and examples to develop MIDP-compliant applications. 
The application was later tested using a JAVA-enabled mobile phone on a GSM network using a GPRSenabled SIM card. The MIDP application is packaged inside a JAVA archive (JAR) file, which contains the applications classes and resource files. This JAR file is the one that actually is downloaded to the physical device (mobile phone) along with the JAVA application descriptor file when an expert wants to use our this prototype.

\subsection{Server}

The server is the main side of the linguistic GDM prototype. It implements the four main modules and the database that stores the problem data as well as problem parameters and the information generated during the decision process. The communication with the client to receive/send information from/to the experts is supported by mobile Internet (M-Internet) technologies (see Figure 9). Concretely, the four modules of the server are:

\section{SERVER}

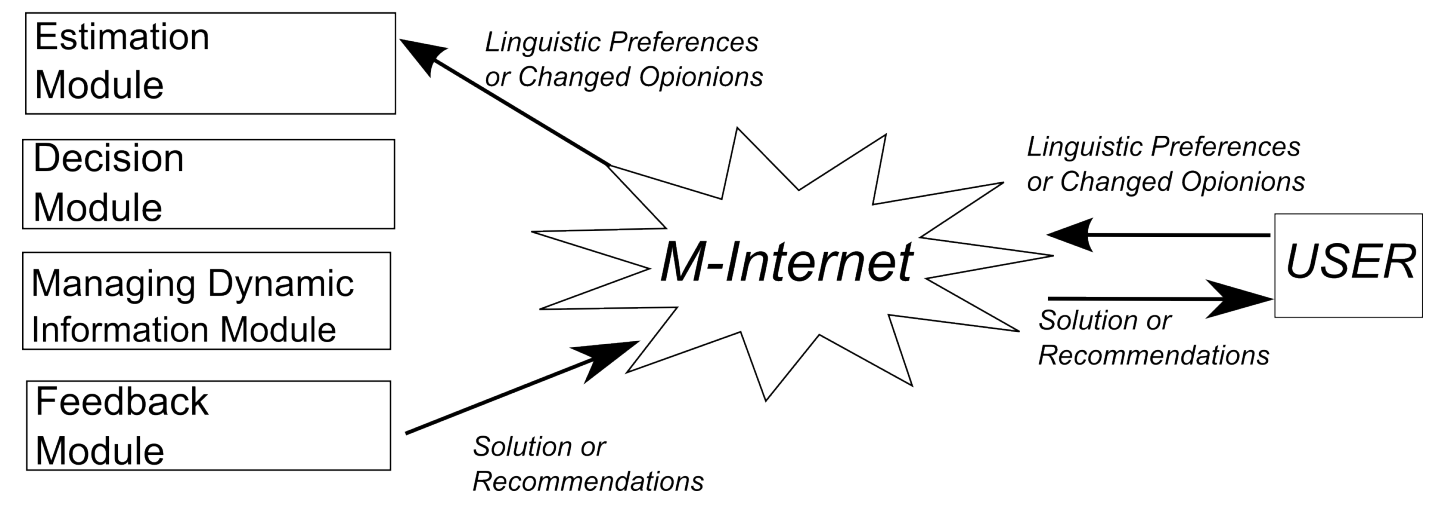

Figure 9: Server modules

1. An estimation module:

This module completes experts' preferences by using that procedure to estimate missing values presented in section 3.1.

\section{A decision module:}

Once the incomplete FLPR are completed, the server starts the decision module to obtain a temporary solution of the problem. In this module the consensus measures are also calculated. If the consensus level has reached the minimum consensus level, the decision process stops and this temporary collective solution is assumed as the final consensus solution. In other case, the decision process should continue.

\section{A managing module of dynamic information:}

If some external factors change during the decision process, any information of the problem could change too. This module is able to manage the changes in the alternatives set by replacing a bad alternative when it does not deserve to be part of the discussion subset, or including a new alternative dynamically generated during the decision process. To do that, the server asks experts if they agree to change the worst alternatives incorporating the new ones in the process. If the majority of the experts accept the change, the module updates the discussion subset of alternatives.

\section{A feedback module:}

When a consensus round is finished without reaching the minimum consensus level, the server starts the feedback module, that calculates the proximity measures and generates recommendations rules. These 
rules help experts to change their preferences about some alternatives to reach consensus. Moreover, some rules are provided to complete the preferences in the cases where missing values exist.

Between client and server some communication functions are developed. In what follows, we present how the modules are connected together with the database, and the order in which each of them is executed.

0. Initialization: An initial step is to insert in the database all the initial parameters of the linguistic GDM problem.

1. Verify user messages and store the main information: When an expert wants to access to the system, he has to send a message through M-Internet using his/her mobile device. The user can send two kinds of messages:

i) A preferences message: It is composed by authentication information (login and password) and his/her preferences about the problem, using a set of labels to represent a FLPR.

ii) A change of alternatives message: It is composed by authentication information (login and password) and his/her linguistic level of agreement with the proposed change of alternatives.

These messages are verified by the server, checking the login and password in the database. If the authentication process is correct, the rest of the information of the message is stored in the database and the server decides if the consensus stage should start (if all experts have provided their preferences) or, if the managing module of dynamic information can be finished (if enough experts provide their agreement degrees on the proposed change of alternatives).

2. Estimate missing values: The server estimates the missing values in the FLPR by following the procedure presented in section 3.1. The server stores this information in the database.

3. Calculate the set of solution alternatives and the consensus measures: The decision module returns the solution set of alternatives in each stage of the decision process. All the information about the temporary solution is saved in the database.

4. Control the consensus state: In this step, the server determines if the required agreement degree has been reached (and thus, the decision process must be finished) or if we must begin a new round of consensus using the feedback mechanism that generates recommendations to change the experts' preferences.

5. Management of new alternatives: When the minimum consensus level has not been reached, the system checks if some new good alternatives appear in the problem environment or an old alternative deserves be removed.

6. Generate the recommendations: In this step, the server calculates the proximity measures and generates the recommendations to change and complete the FLPRs. It sends a message to the experts advising that they can use the software again for reading the recommendations and in such a way to start a new consensus stage. In order to avoid that the collective solution does not converge after several discussion rounds, the prototype stops if the number of rounds surpass MAXCYCLES. These recommendations are saved in the database and sent to the experts through M-Internet.

In the next section we present a practical example on the use of the prototype to provide more detail about its operation.

\section{Example}

Medical diagnosis is an example of decision process that can beneficiate by the use of our system. This scenario presents all the characteristics to be a GDM problem. There is a patient who presents some symptoms, but all of them are common to several diseases. These diseases shape the set of alternatives 
of the problem. In addition, there are some doctors considered specialists in differential diagnosis. They conform the set of experts of the problem and they have to jointly diagnose which is the disease that the patient has contracted. The experts live in different cities of the world and they can not have a meeting to discuss and reach the consensual solution. Moreover, this environment is dynamic in the sense that the patient is now in a hospital and, at any moment, he could present new symptoms or he could set better due to the medication, and thus, any change of state of the patient might be taken into account by the doctors. So, the experts might decide to use our system because they can use the mobile communication technologies to reach the consensus, and they can change some possible diseases in the discussion set of alternatives according with the current patient's state.

The first step to solve a problem using our prototype is to insert all the initial parameters of the problem (experts, alternatives, thresholds, timing...) in the database. We assume a set of three experts (doctors), $\left\{e_{1}, e_{2}, e_{3}\right\}$, and a set of four alternatives (possible diseases) $\left\{x_{1}, x_{2}, x_{3}, x_{4}\right\}$. The remaining parameters (see table 1 ) are used by the system to obtain the necessary consensus degree among the experts.

\begin{tabular}{|c|c|c|}
\hline Name & Value & Description \\
\hline Ndiseases & 4 & Number of diseases in the discussion subset \\
\hline Nexperts & 3 & Number of experts (doctors) \\
\hline minConsDegree & 0.75 & Minimum consensus level required by the problem \\
\hline minProxDegree & 0.75 & Minimum proximity level required for the experts to be noted to change \\
\hline MAXCYCLES & 4 & Maximum number of iterations of the consensus process \\
\hline maxTime & 12 (hours) & Maximum time of waiting for the experts opinions to change \\
\hline minQGDD & $\mathrm{L}$ & Minimum dominance level that an alternative has to reach to avoid to be changed \\
\hline DSsize & 4 & Discussion subset size \\
\hline
\end{tabular}

Table 1: Initial parameters of the problem

When the initial parameters of the problem are defined, the decision making process starts.

\subsection{First round}

The three experts send their FLPRs using their mobile devices as show the Figure 10 with the next set of seven labels:

$$
S=\left\{s_{0}=N, s_{1}=V L, s_{2}=L, s_{3}=M, s_{4}=H, s_{5}=V H, s_{6}=P\right\} .
$$

where $\mathrm{N}=$ Null, $\mathrm{VL}=$ Very Low, $\mathrm{L}=$ Low, $\mathrm{M}=$ Medium, $\mathrm{H}=$ Hight, $\mathrm{VH}=$ Very Hight and $\mathrm{P}=$ Perfect.

\subsubsection{Estimation process}

Two of the experts give incomplete FLPRs $\left\{P^{1}, P^{3}\right\}$. Then, the estimation process completes the missing values in the following steps:

Step 1: The set of elements that can be estimated are:

$$
E M V_{1}^{1}=\{(1,4),(2,3),(3,2),(4,1)\} .
$$

With these estimated preference degrees we have:

$$
P^{1}=\left(\begin{array}{cccc}
- & x & \mathbf{L} & L \\
x & - & V L & \mathbf{V L} \\
\mathbf{V H} & H & - & \mathbf{M} \\
V H & \mathbf{H} & \mathbf{M} & -
\end{array}\right)
$$

As an example, to estimate $p_{14}^{1}$ the procedure is as follows: 


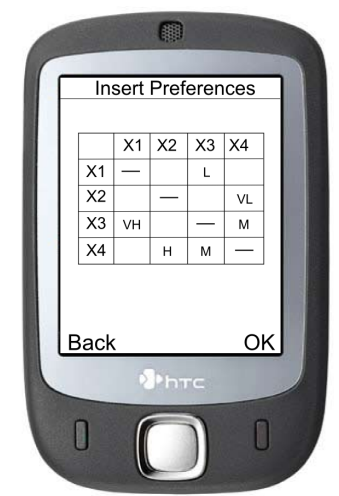

(a) Expert 1

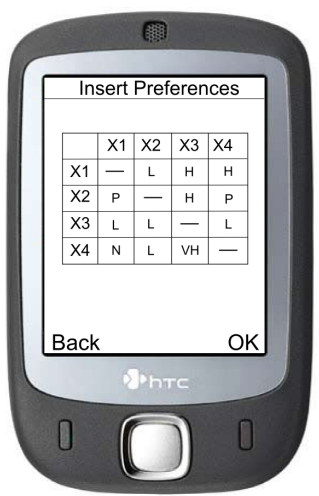

(b) Expert 2

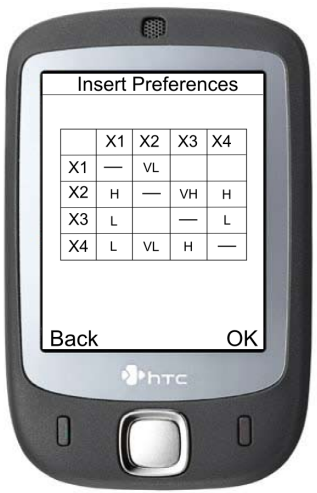

(c) Expert 3

Figure 10: Expert Preferences

$$
\begin{aligned}
& H_{14}^{11}=\{3\} \Rightarrow\left(c p_{14}^{1}\right)^{1}=I\left(p_{13}^{1}\right)+I\left(p_{34}^{1}\right)-I\left(s_{g / 2}\right)=2+3-3=2 . \\
& H_{14}^{12}=\{3\} \Rightarrow\left(c p_{14}^{1}\right)^{2}=I\left(p_{34}^{1}\right)-I\left(p_{31}^{1}\right)+I\left(s_{g / 2}\right)=3-5+3=1 . \\
& H_{14}^{13}=\{3\} \Rightarrow\left(c p_{14}^{1}\right)^{3}=I\left(p_{13}^{1}\right)-I\left(p_{43}^{1}\right)+I\left(s_{g / 2}\right)=2-3+3=2 . \\
& \mathcal{K}=3 \Rightarrow c p_{14}^{1}=L \text { given that round }\left(\frac{\left(c p_{14}^{1}\right)^{1}+\left(c p_{14}^{1}\right)^{2}+\left(c p_{14}^{1}\right)^{3}}{3}\right)=1,67
\end{aligned}
$$

Step 2: The set of elements that can be estimated are:

$$
E M V_{2}^{1}=\{(1,2),(2,1)\}
$$

After these elements have been estimated, we have the following complete FLPR:

$$
\bar{P}^{1}=\left(\begin{array}{cccc}
- & M & \mathbf{L} & L \\
M & - & V L & \mathbf{V L} \\
\mathbf{V H} & H & - & \mathbf{M} \\
V H & \mathbf{H} & \mathbf{M} & -
\end{array}\right) .
$$

For $P^{3}$ we get the following complete FLPR:

$$
\bar{P}^{3}=\left(\begin{array}{cccc}
- & \mathbf{V L} & H & M \\
\mathbf{H} & - & \mathbf{V H} & \mathbf{H} \\
\mathbf{L} & N & - & L \\
\mathbf{L} & \mathbf{V L} & \mathbf{H} & -
\end{array}\right) .
$$

\subsubsection{Selection process}

In this phase the system obtains the collective temporary solution by aggregating the experts' preferences.

1. Aggregation:

Once the incomplete FLPRs are completed, we aggregate them by means of the LOWA operator. We use the linguistic quantifier most of defined as $Q(r)=r^{1 / 2}$. Then, we obtain the following collective FLPR:

$$
P^{c}=\left(\begin{array}{cccc}
- & L & H & M \\
V H & - & H & V H \\
H & M & - & M \\
M & M & H & -
\end{array}\right)
$$




\section{Exploitation:}

Using again the same linguistic quantifier "most of", we obtain $Q G D D_{i}$ and $Q G N D D_{i} \forall x_{i} \in X$ :

Table 2: Choice degrees

\begin{tabular}{|c|c|c|c|c|}
\hline & $x_{1}$ & $x_{2}$ & $x_{3}$ & $x_{4}$ \\
\hline$Q G D D_{i}$ & $\mathrm{M}$ & $\mathrm{VH}$ & $\mathrm{H}$ & $\mathrm{H}$ \\
\hline$Q G N D D_{i}$ & $\mathrm{VH}$ & $\mathrm{P}$ & $\mathrm{P}$ & $\mathrm{P}$ \\
\hline
\end{tabular}

and, the maximal sets are:

$$
X^{Q G D D}=\left\{x_{2}\right\} \text { and } X^{Q G N D D}=\left\{x_{2}\right\} .
$$

\subsubsection{Consensus process}

In this phase the system calculates the consensus measures.

1. Similarity matrices:

$$
\begin{aligned}
S M_{12} & =\left(\begin{array}{cccc}
- & 0.83 & 0.66 & 0.66 \\
0.50 & - & 0.50 & 0.16 \\
0.50 & 0.66 & - & 0.83 \\
0.16 & 0.66 & 0.66 & -
\end{array}\right) \\
S M_{13} & =\left(\begin{array}{cccc}
- & 0.66 & 0.66 & 0.83 \\
0.83 & - & 0.33 & 0.50 \\
0.50 & 0.33 & - & 0.83 \\
0.50 & 0.50 & 0.83 & -
\end{array}\right) \\
S M_{23} & =\left(\begin{array}{cccc}
- & 0.83 & 1.00 & 0.83 \\
0.66 & - & 0.83 & 0.66 \\
1.00 & 0.66 & - & 1.00 \\
0.66 & 0.83 & 0.83 & -
\end{array}\right)
\end{aligned}
$$

2. Consensus matrix:

$$
C M=\left(\begin{array}{cccc}
- & 0.77 & 0.77 & 0.77 \\
0.66 & - & 0.55 & 0.44 \\
0.66 & 0.55 & - & 0.88 \\
0.44 & 0.66 & 0.77 & -
\end{array}\right)
$$

3. Consensus degrees on pairs of alternatives. The element $(l, k)$ of $C M$ represents the consensus degrees on the pair of alternatives $\left(x_{l}, x_{k}\right)$.

4. Consensus on alternatives:

$$
c a^{1}=0.77 \quad c a^{2}=0.55 \quad c a^{3}=0.69 \quad c a^{4}=0.62
$$

5. Consensus on the relation:

$$
c r=0.66
$$

As $c r<\operatorname{minConsDegree~}=0.75$ is satisfaid, then it is concluded that there is no consensus amongst the experts, and consequently, the system should to continue by executing the next two processes: managing process of dynamic information to replace some alternatives in the discussion subset and feedback process to support the experts' changes in their preferences in order to increase $\mathrm{cr}$. 


\subsubsection{Managing process of dynamic information}

As soon as the system has verified that the minimum consensus level among the experts has not been reached and before beginning a new round of consensus, it is necessary to update all the information of the problem that there could be changed during the process.

In this case, the patient, due to the medication, has started to show a new symptom that is typical of a disease that was not included in the initial discussion subset of the problem. This new situation does not suppose any problem because the system manages the dynamic information. We identify those alternatives with low choice degrees and ask the experts if they agree to replace those identified alternatives by others new ones that has appeared (See Figure 11a).

The experts' answers were the following: (Agree, Nor Agree/Nor Disagree and Completely Agree). The system applies the LOWA operator to aggregate these opinions and obtain a collective agreement degree. In this case we obtain ,(Agree), what represents an affirmative position to introduce the changes of alternatives. Therefore, the change of $x_{1}$ by $x_{5}$ is done. The experts will be informed about it and then they are urged to refill their preferences about the new alternative.

\subsubsection{Feedback process}

\section{- Computation of proximity measures:}

1. Proximity matrices:

$$
\begin{aligned}
P M_{1} & =\left(\begin{array}{cccc}
- & 0.83 & 0.66 & 0.83 \\
0.66 & - & 0.50 & 0.33 \\
0.83 & 0.83 & - & 1.00 \\
0.66 & 0.83 & 0.83 & -
\end{array}\right) \\
P M_{2} & =\left(\begin{array}{cccc}
- & 1.00 & 1.00 & 0.83 \\
0.83 & - & 1.00 & 0.83 \\
0.66 & 0.83 & - & 0.83 \\
0.50 & 0.83 & 0.83 & -
\end{array}\right) \\
P M_{3} & =\left(\begin{array}{cccc}
- & 0.83 & 1.00 & 1.00 \\
0.83 & - & 0.83 & 0.83 \\
0.66 & 0.50 & - & 0.83 \\
0.83 & 0.66 & 1.00 & -
\end{array}\right)
\end{aligned}
$$

2. Proximity on pairs of alternatives: $P P_{i}=P M_{i}$.

3. Proximity on alternatives (See Table 3):

Table 3: Proximity measures on alternatives

\begin{tabular}{|c|c|c|c|}
\hline$x_{1}$ & $x_{2}$ & $x_{3}$ & $x_{4}$ \\
\hline$p a_{1}^{1}=0.77$ & $p a_{1}^{2}=0.50$ & $p a_{1}^{3}=0.88$ & $p a_{1}^{4}=0.77$ \\
\hline$p a_{2}^{1}=0.94$ & $p a_{2}^{2}=0.88$ & $p a_{2}^{3}=0.77$ & $p a_{2}^{4}=0.72$ \\
\hline$p a_{3}^{1}=0.94$ & $p a_{3}^{2}=0.83$ & $p a_{3}^{3}=0.66$ & $p a_{3}^{4}=0.83$ \\
\hline
\end{tabular}


4. Proximity on the relation:

$$
p r_{1}=0.73 \quad p r_{2}=0.83 \quad p r_{3}=0.81
$$

\section{- Production of advice:}

1. Identification phase:

(a) Identification of experts:

$$
E X P C H=\left\{e_{i} \mid p r_{i}<\operatorname{minProxDegree}\right\}=\left\{e_{1}\right\}
$$

(b) Identification of alternatives:

$$
\begin{aligned}
& A L T_{1}=\left\{x_{l} \in X \mid p a_{l}^{i}<\operatorname{minProxDegree} \wedge e_{i} \in E X P C H\right\}= \\
& \left\{x_{2}\right\}
\end{aligned}
$$

(c) Identification of pairs of alternatives to generate recomendations:

$$
P A L T_{1}=\left\{\left(x_{2}, x_{1}\right),\left(x_{2}, x_{3}\right),\left(x_{2}, x_{4}\right)\right\}
$$

2. Recommendation phase:

In this phase, we have to take into account that alternative $x_{1}$ has been replaced in the previous process by $x_{5}$. So, $x_{1}$ does not need rules to be modified and there is a new alternative in the discussion subset, $x_{5}$, that needs new preference values. The recommendations interface is shown in Figure 11b.

(a) Rules to change the opinions:

- Because $x_{1}$ has been replaced, $p_{21}^{1}$ does not need be modified.

- Because $p_{23}^{1}$ is an estimated element, the expert can not modify his preference.

- Because $p_{24}^{1}<p_{24}^{c}$, expert $e_{1}$ is advised to increase the assessment of this preference value.

(b) Rules to complete the missing values:

- Expert $e_{1}$ is advised to provide a value for $p_{23}^{1}$ near to $V L$

- Expert $e_{1}$ is advised to provide a value for $p_{32}^{1}$ near to $H$

- Expert $e_{3}$ is advised to provide a value for $p_{32}^{3}$ near to $N$

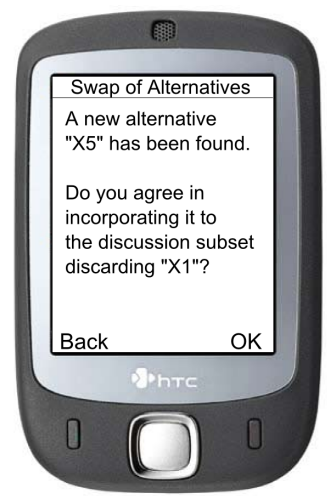

(a) Swap of alternatives

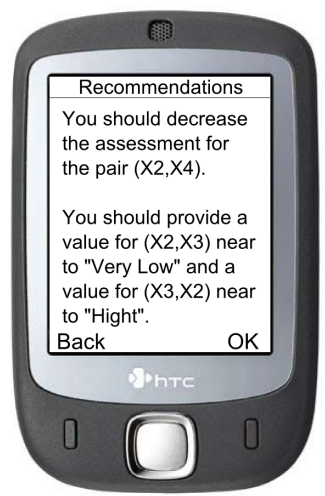

(b) Recommendations

Figure 11 


\subsection{Second round}

The experts send their preferences about the new discussion subset to start the second round (see Figure $12)$.

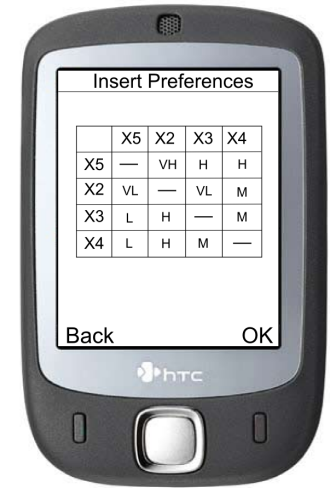

(a) Expert 1

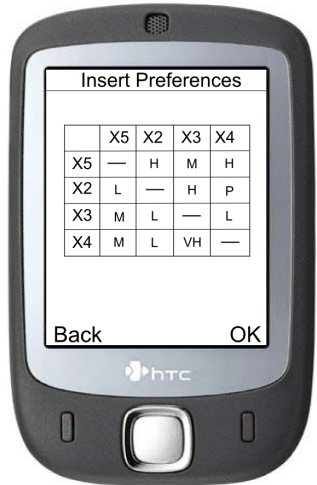

(b) Expert 2

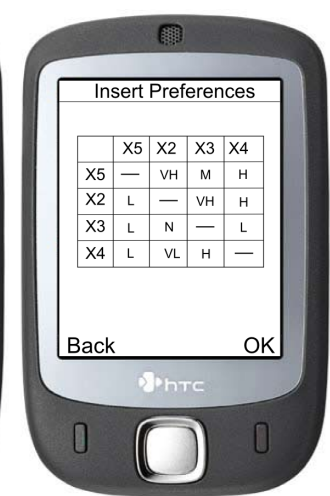

(c) Expert 3

Figure 12: Expert Preferences

As the experts have inserted complete preference relations, the estimation missing values process is avoided and the system continues the decision process.

\subsubsection{Selection process}

1. Aggregation:

The collective FLPR is:

$$
P^{c}=\left(\begin{array}{cccc}
- & V H & H & H \\
L & - & H & V H \\
M & M & - & M \\
M & M & H & -
\end{array}\right)
$$

2. Exploitation:

Using again the same linguistic quantifier "most of", we obtain the following choice degrees: q

Table 4: Choice degrees in 2nd round

\begin{tabular}{|c|c|c|c|c|}
\hline & $x_{5}$ & $x_{2}$ & $x_{3}$ & $x_{4}$ \\
\hline$Q G D D_{i}$ & $\mathrm{VH}$ & $\mathrm{H}$ & $\mathrm{M}$ & $\mathrm{H}$ \\
\hline$Q G N D D_{i}$ & $\mathrm{P}$ & $\mathrm{VH}$ & $\mathrm{VH}$ & $\mathrm{VH}$ \\
\hline
\end{tabular}

Clearly, the maximal sets are:

$$
X^{Q G D D}=\left\{x_{5}\right\} \text { and } X^{Q G N D D}=\left\{x_{5}\right\}
$$

\subsubsection{Consensus process}

Consensus on the relation:

$$
c r=0.79
$$

Because $c r>$ minConsDegree, then it is concluded that there is the required consensus amongst the experts, and consequently, the current solution is the final solution, that is stored and sent to the experts (see Figure 13). 


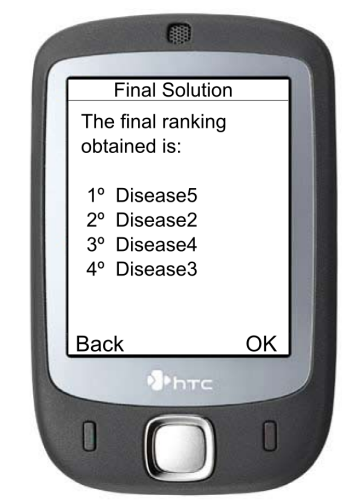

Figure 13: Final solution

\section{Conclusions}

We have presented a new model of linguistic GDM based on dynamic information and mobile technologies. We have also implemented a prototype of this system. It is designed to deal with linguistic GDM problems based on dynamic sets of alternatives, which uses the advantages of mobile Internet technologies to improve the user-system interaction through decision process. The experts can use FLPR to express their preferences and it provides a tool that manages lack of information when an expert is not able to give a complete FLPR. We have used mobile phones as the device used by the experts to send their FLPRs but the structure of the prototype is designed to use any other mobile device as PDAs.

Shortly, with this new GDM model we shall be able to model linguistic GDM problems in which experts could interact in anywhere and anytime, quickly, in a flexible way, and dynamic frameworks.

\section{Acknowledgment}

This paper has been developed with the financing of FEDER funds in FUZZYLING project TIN2007-61079, FUZZYLING-II project TIN2010-17876, PETRI project PET2007-0460, Andalusian Excellence project TIC05299, and project of Ministry of Public Works 90/07.

\section{References}

[1] http://java.sun.com/products/midp/.

[2] http://java.sun.com/products/sjwtoolkit/.

[3] S. Alonso, F.J. Cabrerizo, F. Chiclana, F. Herrera, and E. Herrera-Viedma. Group decision-making with incomplete fuzzy linguistic preference relations. International Journal of Intelligent Systems, 24(2):201$222,2009$.

[4] S. Alonso, E. Herrera-Viedma, F. Chiclana, and F.Herrera. Individual and social strategies to deal with ignorance situations in multi-person decision making. International Journal of Information Technology \& Decision Making, 8(2):313-333, 2009.

[5] B. Arfi. Fuzzy decision making in politics: A linguistic fuzzy-set approach. Political Analysis, 13(1):23$56,2005$.

[6] J. Aronson, T. Liang, and E. Turban. Decision support systems and intelligent systems. Pearson, Upper Saddle River, 2005. 
[7] D. Ben-Arieh and Z. Chen. Linguistic-labels aggregation and consensus measure for autocratic decision making using group recommendations. IEEE Transactions on Systems, Man, and Cybernetics. Part A: Systems and Humans, 36(3):558-568, 2006.

[8] F. E. Boran, S. Gen, M. Kurt, and D. Akay. A multi-criteria intuitionistic fuzzy group decision making for supplier selection with topsis method. Expert Systems with Applications, 36(8):11363-11368, 2009.

[9] G. Bordogna and G. Pasi. An ordinal information retrieval model. International Journal of Uncertainty, Fuzziness and Knowledge-Based Systems, 9:63-76, 2001.

[10] S. Burigat and L. Chittaro. Interactive visual analysis of geographic data on mobile devices based on dynamic queries. Journal of Visual Languages and Computing, 19:99-122, 2008.

[11] F.J. Cabrerizo, S. Alonso, and E. Herrera-Viedma. A consensus model for group decision making problems with unbalanced fuzzy linguistic information. International Journal of Information Technology \& Decision Making, 8(1):109-131, 2009.

[12] F.J. Cabrerizo, J. Lpez-Gijón, A.A. Ruíz-Rodríguez, and E. Herrera-Viedma. A model based on fuzzy linguistic information to evaluate the quality of digital libraries. International Journal of Information Technology \& Decision Making, 9(3):455-472, 2010.

[13] F.J. Cabrerizo, J.M. Moreno, I.J. Pérez, and E. Herrera-Viedma. Analyzing consensus approaches in fuzzy group decision making: advantages and drawbacks. Soft Computing, 14(5):451-463, 2010.

[14] F.J. Cabrerizo, I.J. Pérez, and E. Herrera-Viedma. Managing the consensus in group decision making in an unbalanced fuzzy linguistic context with incomplete information. Knowledge-Based Systems, 23(2):169-181, 2010.

[15] F. Chiclana, F. Herrera, and E. Herrera-Viedma. Integrating three representation models in fuzzy multipurpose decision making based on fuzzy preference relations. Fuzzy Sets and Systems, 97(1):3348, 1998.

[16] H. Clarke. Classical decision rules and adaptation to climate change. The Australian Journal of Agricultural and Resource Economics, 52:487-504, 2008.

[17] J. Cowie and F. Burstein. Quality of data model for supporting mobile decision making. Decision Support Systems, 43:1675-1683, 2007.

[18] S. Daume and D. Robertson. An architecture for the deployment of mobile decision support systems. Expert Systems with Applications, 19:305-318, 2000.

[19] H. Dia. An agent-based approach to modelling driver route choice behaviour under the influence of real-time information. Transportation Research Part C, 10:331-349, 2002.

[20] A. Eren, A. Subasi, and O. Coskun. A decision support system for telemedicine through the mobile telecommunications platform. Journal of Medical Systems, 32(1), 2008.

[21] J. Fodors and M. Roubens. Fuzzy preference modelling and multicriteria decision support. Kluwer Academic Publishers, Dordrecht, 1994.

[22] F. Herrera and E. Herrera-Viedma. Aggregation operators for linguistic weighted information. IEEE Transactions on Systems, Man, and Cybernetics. Part A: Systems and Humans, 27:646-656, 1997.

[23] F. Herrera and E. Herrera-Viedma. Choice functions and mechanisms for linguistic preference relations. European Journal of Operational Research, 120:144-161, 2000.

[24] F. Herrera and E. Herrera-Viedma. Linguistic decision analisis: steps for solving decision problems under linguistic information. Fuzzy Set and Systems, 115:67-82, 2000. 
[25] F. Herrera, E. Herrera-Viedma, and L. Martínez. A fuzzy linguistic methodology to deal with unbalanced linguistic term sets. IEEE Transactions on Fuzzy Systems, 16(2):354-370, 2008.

[26] F. Herrera, E. Herrera-Viedma, and J.L. Verdegay. A sequential selection process in group decision making with a linguistic assessment approach. Information Sciences, 85(4):223-239, 1995.

[27] F. Herrera, E. Herrera-Viedma, and J.L. Verdegay. Direct approach processes in group decision making using linguistic owa operators. Fuzzy Sets and Systems, 79:175-190, 1996.

[28] F. Herrera, E. Herrera-Viedma, and J.L. Verdegay. A model of consensus in group decision making under linguistic assessments. Fuzzy Sets and Systems, 78(1):73-87, 1996.

[29] F. Herrera, E. Herrera-Viedma, and J.L. Verdegay. Linguistic measures based on fuzzy coincidence for reaching consensus in group decision making. International Journal of Approximate Reasoning, 16:309-334, 1997.

[30] E. Herrera-Viedma. An information retrieval model with ordinal linguistic weighted queries based on two weighting elements. International Journal of Uncertainty, Fuzziness and Knowledge-Based Systems, 9:77-88, 2001.

[31] E. Herrera-Viedma, S. Alonso, F. Chiclana, and F. Herrera. A consensus model for group decision making with incomplete fuzzy preference relations. IEEE Transactions on Fuzzy Systems, 15(5):863$877,2007$.

[32] E. Herrera-Viedma, F. Chiclana, F. Herrera, and S. Alonso. A group decision-making model with incomplete fuzzy preference relations based on additive consistency. IEEE Transactions on Systems, Man, and Cybernetics. Part B, Cybernetics, 37(1):176-189, 2007.

[33] E. Herrera-Viedma, F. Herrera, and F. Chiclana. A consensus model for multiperson decision making with different preference structures. IEEE Transactions on Systems, Man, and Cybernetics. Part A: Systems and Humans, 32(3):394-402, 2002.

[34] E. Herrera-Viedma, F. Herrera, F. Chiclana, and M.Luque. Some issues on consistency of fuzzy preference relations. European Journal of Operational Research, 154(1):98-109, 2004.

[35] E. Herrera-Viedma and A. G. López-Herrera. A model of information retrieval system with unbalanced fuzzy linguistic information. International Journal of Intelligent Systems, 22(11):1197-1214, 2007.

[36] E. Herrera-Viedma, L. Martinez, F. Mata, and F. Chiclana. A consensus support system model for group decision-making problems with multi-granular linguistic preference relations structures. IEEE Trans. on Fuzzy Systems, 13(5):644-658, 2005.

[37] E. Herrera-Viedma, G. Pasi, A. G. López-Herrera, and C. Porcel. Evaluating the information quality of web sites: A methodology based on fuzzy. Journal of the American Society for Information Science and Technology, 57(4):538-549, 2006.

[38] E. Herrera-Viedma and E. Peis. Evaluating the informative quality of documents in SGML-format using fuzzy linguistic techniques based on computing with words. Information Processing 8 Management, 39(2):195-213, 2003.

[39] E. Herrera-Viedma, E. Peis, J.M. Morales, S. Alonso, and K. Anaya. A fuzzy linguistic model to evaluate the quality of web sites that store xml documents. International Journal of Approximate Reasoning, 46(1):226-253, 2007.

[40] T. Imielinski and B.R. Badrinath. Mobile wireless computing: challenges in data management. Communications of the ACM, 37(10):18-28, 1994. 
[41] J. Kacprzyk and M. Fedrizzi. Multiperson decision making models using fuzzy sets and possibility theory. Kluwer Academic Publishers, Dordrecht, 1990.

[42] J. Katz. Handbook of Mobile Communication Studies. The MIT Press, 2008.

[43] N. Kim, H. Seok, K. Joo, and J. Young. Context-aware mobile service for routing the fastest subway path. Expert Systems with Applications, 36:3319-3326, 2009.

[44] J. López-Gijón, B. Avila, I.J. Pérez, and E. Herrera-Viedma. La calidad en las bibliotecas universitarias biomédicas según sus usuarios. El Profesional de la Información, 19(3):255-259, 2010.

[45] F. Mata, L. Martínez, and E. Herrera-Viedma. An adaptive consensus support model for group decision making problems in a multi-granular fuzzy linguistic context. IEEE Transactions on Fuzzy Systems, 17(2):279-290, 2009.

[46] J. Muntermann. Event-Driven mobile Finantial Information Services: Mobile notification and Decision Support for private investors. DUV, 2008.

[47] F. Navarro, A. Schulter, F. Koch, M. Assunao, and C.B. Westphall. Grid middleware for mobile decision support system. Proceedings of the International Conference on Networking, International Conference on Systems and International Conference on Mobile Communications (ICNICONSMLC'06), 2006.

[48] I.J. Pérez, F.J. Cabrerizo, and E. Herrera-Viedma. A mobile decision support system for dynamic group decision making problems. IEEE Transactions on Systems, Man and Cybernetics - Part A: Systems and Humans, doi:10.1109/TSMCA.2010.2046732, 2010, in press.

[49] G. Pistolesi. Intelligent Decision-making Support Systems: Foundations, Applications and Challenges (cap 13). Springer, 2006.

[50] C. Porcel and E. Herrera-Viedma. Dealing with incomplete information in a fuzzy linguistic recommender system to disseminate information in university digital libraries. Knowledge-Based Systems, $23: 32-39,2010$.

[51] C. Porcel, A.G. Lopez-Herrera, and E. Herrera-Viedma. A recommender system for research resources based on fuzzy linguistic modeling. Expert Systems with Applications, 36(3):15173-5183, 2009.

[52] C. Porcel, J.M. Moreno, and E. Herrera-Viedma. A multi-disciplinar recommender system to advice research resources in university digital libraries. Expert Systems with Applications, 36(10):12520-12528, 2009.

[53] Th.L. Saaty. Time dependent decision-making; dynamic priorities in the AHP/ANP: Generalizing from points to functions and from real to complex variables. Mathematical and Computer Modelling, 46:860$891,2007$.

[54] A. Sanayei, S. F. Mousavi, and A. Yazdankhah. Group decision making process for supplier selection with vikor under fuzzy environment. Expert Systems with Applications, 37(1):24-30, 2010.

[55] J. Schiller. Mobile Communications (2nd Edition). Addison Wesley, 2003.

[56] Flora S. Tsai, W. Han, J. Xu, and H. C. Chua. Design and development of a mobile peer-to-peer social networking application. Expert Systems with Applications, 36(8):11077-11087, 2009.

[57] U. Varsney and R. Vetter. Emerging mobile and wireless networks. Communications of the ACM, $43(6): 73-81,2000$.

[58] W. Wen, Y.H. Chen, and H.H. Pao. A mobile knowledge management decision support system for automatically conducting an electronic business. Knowledge-Based Systems, 21(7), 2008. 
[59] Z.S. Xu. Dynamic linguistic preference relations and their use in multi-period decision making. International Conference on Management Science and Engineering, Harbin, P.R.China, pages 345-350, 2007.

[60] L.A. Zadeh. The concept of a linguistic variable and its applications to approximate reasoning. Information Sciences, Part I, II, III, 8,8,9:199-249,301-357,43-80, 1975.

[61] Z. Zhang and X. Chu. Fuzzy group decision-making for multi-format and multi-granularity linguistic judgments in quality function deployment. Expert Systems with Applications, 36(5):9150-9158, 2009. 